\title{
Nanocrystalline silicon prepared at high growth rate using helium dilution
}

\author{
KOYEL BHATTACHARYA and DEBAJYOTI DAS* \\ Energy Research Unit, Indian Association for the Cultivation of Science, Kolkata 700 032, India
}

\begin{abstract}
Growth and optimization of the nanocrystalline silicon (nc-Si : $\mathrm{H}$ ) films have been studied by varying the electrical power applied to the helium diluted silane plasma in RF glow discharge. Wide optical gap and conducting intrinsic nanocrystalline silicon network of controlled crystalline volume fraction and oriented crystallographic lattice planes have been obtained at a reasonably high growth rate from helium diluted silane plasma, without using hydrogen. Improving crystallinity in the network comprising $\sim 10 \mathrm{~nm}$ Si-nanocrystallites and contributing optical gap widening, conductivity ascending and that obtained during simultaneous escalation of the deposition rate, promises significant technological impact.
\end{abstract}

Keywords. Nanocrystalline silicon; helium dilution; wide optical gap; RF glow discharge.

\section{Introduction}

General interest in the nano/micro-crystalline silicon materials processing has been increasing significantly because of its enormously promising utilization in thin film technologies such as solar cells and thin film transistors. Microcrystalline silicon films have higher doping efficiency, higher carrier mobility and lower optical absorption compared to their amorphous counterparts. Because of the favourable combination of all these properties, $\mu \mathrm{c}-\mathrm{Si}: \mathrm{H}$ films are being used as a window layer and also at the tunnel junction in $a$-Si based solar cells, as well as a carrier injection layer in thin film transistors (Uchida et al 1986). Fabrication of silicon solar cells with entirely nano/ micro-crystalline components have also been reported with marked improvement in the near-infrared absorption of the solar spectrum and in possible absence of light induced degradation (Prasad et al 1991).

Nanocrystalline-Si (nc-Si) could realize quantum dots and quantum ball structures where zero dimensional quantum confinement effects on electrons and holes reveal nonlinearities in optical properties. Nano-Si films exhibit optical gap widening (Furukawa and Miyasato 1988; Das 2003), photoluminescence (Takagi et al 1990; Liu et al 1994; Mangolini et al 2005; Sankaran et al 2005), electroluminescence (Dehlinger et al 2000) and resonant tunneling (Fortunate et al 1989), as a consequence of three dimensionally confined quantum size effect. Photoluminescence quantum yield exceeding $60 \%$ has been reported by recent investigations (Jurbergs et al 2006). Nano-Si thin films appear to be promising for future applications in quantum electronics and quantum optics, in addition to its potential use in ultra large-scale integrated circuit

*Author for correspondence (erdd@iacs.res.in)
(ULSIC) (One et al 1993) and large area electronic devices (Roca i Cabarrocas et al 2007).

High $\mathrm{H}_{2}$ dilution and high level of electrical excitation to the $\mathrm{SiH}_{4}$ plasma are the two critical parameters facilitating the growth of nanocrystalline network in plasma enhanced chemical vapour deposition (PECVD). However, high $\mathrm{H}_{2}$ dilution retards the film growth rate and high electrical power causes surface damage of other component layers in the device. The combination of stringent parameters required for its growth limits the use of this material in device fabrication. Several approaches have been attempted to increase the deposition rate by using highdensity plasma sources e.g. microwave, very high frequency, and electron-cyclotron resonance plasmas, with their individual merits and demerits. Noble gas (e.g. He, $\mathrm{Ar}, \mathrm{Xe}$ ) dilution to $\mathrm{SiH}_{4}$ is one way of producing high density plasma at low electrical excitation to increase the deposition rate. However, high Ar dilution leads to columnar growth and mostly produce defective network (Knights 1980; Das et al 2001), while Xe dilution maintains an amorphous nature of the network even at a very high RF power applied to the plasma (Matsuda et al 1991). He dilution undoubtedly increases the growth rate, however, it has not been reported to produce nanocrystalline silicon network (Tchakarov et al 2004). The present article deals with the development of nanocrystalline silicon network at high growth rate from purely helium diluted silane plasma, without hydrogen dilution, at moderate temperature compatible for device fabrication.

\section{Experimental}

Hydrogenated nanocrystalline silicon (nc-Si : H) films were prepared in a capacitively coupled RF $(13.56 \mathrm{MHz})$ glow discharge system at a substrate temperature of $200^{\circ} \mathrm{C}$, 
using $2 \mathrm{sccm} \mathrm{SiH}_{4}$ as the source gas and $70 \mathrm{sccm} \mathrm{He}$ as the only diluent in the plasma. The gas pressure in the reaction chamber was maintained at 0.5 Torr by controlling the mechanical throttle valve placed before the boosterrotary combination. The electrical power applied to the electrodes in the reactor was the only parameter that was varied from $40-120 \mathrm{~W}$ in preparing a set of films. The nc-Si : H films were deposited on glass substrates for the study of electrical and optical properties. The structural characterization was done using infrared vibrational spectroscopy on films deposited on polished surface of single crystal Si wafers. Raman studies as well as X-ray diffraction were performed with samples on glass substrates. For transmission electron microscopic (TEM) studies, samples were deposited on carbon coated copper microscope grids. Scanning electron microscopy (SEM) was carried out with samples on glass overcoated with $\sim 100 \AA$ of Pt to avoid charging effects. The thickness of the films was maintained between 650 and $850 \mathrm{~nm}$, as measured by Dektak 6M surface profiler.

\section{Results and discussion}

A set of films has been prepared by changing the RF power $(P)$ applied to plasma from $40-120$ Watts. The average film deposition rate $\left(R_{\mathrm{D}}\right)$ as measured by Dektak $6 \mathrm{M}$ surface profiler, has been given in table 1 . It has been observed that the film deposition rate follows a sublinear dependence with $P$, hence produces the $\mathrm{SiH}_{4}$ depleted plasma which could initiate heterogeneity and promote nanocrystallization in the network (Knights 1980; Matsuda 1983; Das et al 2001).

The optical density data of the samples was obtained from the transmission and reflection measurements in the UV-visible region by a double beam spectrophotometer (Hitachi 330, Japan). The absorption coefficient spectra of the films have been presented in figure 1. A gradual reduction in the visible absorption along with a systematic shift of the onset of optical absorption towards higher energy has been pronounced for samples prepared at increasing RF power, which signifies optical gap widening of the network. The magnitude of the optical absorption at $2.3 \mathrm{eV}$ for all the samples has been shown in table 1 , for numerical comparison. To get an overall idea of the optical gap, we have generalized the Tauc's plot $\left[(\alpha h v)^{1 / 2} \propto\right.$ $\left.\left(h v-E_{\mathrm{g}}\right)\right]$ as applicable over the entire network spectrum. By extrapolating the straight portion of the Tauc's plot to $\alpha=0$ at the onset of optical absorption (as shown by the inset of figure 1), the optical gap $\left(E_{\mathrm{g}}\right)$ of the samples was estimated and that shows its gradual widening, as presented in table 1. Systematic reduction in $\alpha$ and simultaneous widening of $E_{\mathrm{g}}$ signify progressive crystallization of the network at enhanced RF power applied to the plasma (Das 1994).

The room-temperature dark conductivity $\left(\sigma_{\mathrm{D}}\right)$ of the samples was measured at a vacuum of $10^{-6}$ Torr after annealing the samples at $150^{\circ} \mathrm{C}$ for $1 \mathrm{~h}$ and using $\mathrm{Al}$ electrodes in gap-cell configuration. The dark conductivity activation energy $\left(E_{\mathrm{a}}\right)$ was estimated from the Arrhenius plot of the conductivity data obtained during cooling of the sample from $150-25^{\circ} \mathrm{C}$. The $\sigma_{\mathrm{D}}$ and its activation energy remained virtually unchanged up to $80 \mathrm{~W}$ of applied $\mathrm{RF}$ power. However, for $P=120 \mathrm{~W}, \sigma_{\mathrm{D}}$ increased by an order of magnitude with simultaneous reduction in $E_{\mathrm{a}}$, as shown in table 1 .

The silicon-hydrogen bonding configurations in the network were investigated from the infrared absorption studies by an FTIR spectrophotometer (Nicolet Magna-IR 750). The $\mathrm{Si}-\mathrm{H}$ bonding structures of the film prepared at $P=40 \mathrm{~W}$ is of dominantly mono-hydride configuration contributing to the stretching mode absorption mostly around $2000 \mathrm{~cm}^{-1}$. The nature of $\mathrm{Si}-\mathrm{H}$ bonding gradually changes from mono-hydride to poly-hydride configuration with increase in $P$, as shown in figure 2 . The spectrum corresponding to the film prepared at $P=120 \mathrm{~W}$ signifies the formation of nanocrystalline structures by its dominant poly-hydride $\left(\left(\mathrm{SiH}_{2}\right)_{n}\right)$ bonding configuration exhibited by the marked increase in relative intensity of IR absorption peak at $\sim 2090 \mathrm{~cm}^{-1}$, along with the reduced integrated intensity of the overall $\mathrm{Si}-\mathrm{H}$ stretching band (Das 2003). The bonded hydrogen content $\left(C_{\mathrm{H}}\right)$ in the films were estimated from the intensity of IR absorption in the wagging mode vibration around $630 \mathrm{~cm}^{-1}$, using

$$
C_{\mathrm{H}}=\left(A_{\omega} / N_{\mathrm{Si}}\right) \int \alpha \cdot d \omega / \omega \times 100 \text { at. } \% \text {, }
$$

where $A_{\omega}=1.6 \times 10^{19} \mathrm{~cm}^{-2}$ is the $\mathrm{Si}-\mathrm{H}$ wagging mode oscillator strength and $N_{\mathrm{Si}}=5 \times 10^{22} \mathrm{~cm}^{-3}$ the atomic density of crystalline $\mathrm{Si}$. The bonded $\mathrm{H}$-content reduces from $24 \cdot 8-13.3$ at $\%$ with the increase in $P$ from $40-120 \mathrm{~W}$, as shown in table 1 .

Table 1. Properties of nc-Si : H films prepared by He-dilution at different RF power.

\begin{tabular}{|c|c|c|c|c|c|c|c|}
\hline $\begin{array}{l}\text { RF power, } \\
P(\mathrm{~W})\end{array}$ & $\begin{array}{c}\text { Deposition rate, } \\
R_{\mathrm{D}}(\AA / \mathrm{min})\end{array}$ & $\begin{array}{c}\text { Absorption } \\
\text { coefficient } \\
\text { at } 2 \cdot 3 \mathrm{eV}, \alpha\left(\mathrm{cm}^{-1}\right)\end{array}$ & $\begin{array}{l}\text { Optical gap, } \\
E_{\mathrm{g}}(\mathrm{eV})\end{array}$ & $\begin{array}{c}\text { Dark } \\
\text { conductivity, } \\
\sigma_{\mathrm{D}}\left(\mathrm{S} \cdot \mathrm{cm}^{-1}\right)\end{array}$ & $\begin{array}{l}\text { Activation } \\
\text { energy, } \\
E_{\mathrm{a}}(\mathrm{meV})\end{array}$ & $\begin{array}{c}\text { H-content, } \\
C_{\mathrm{H}}(\mathrm{at} \%)\end{array}$ & $\begin{array}{c}\text { Crystalline } \\
\text { volume } \\
\text { fraction, } F_{C}(\%)\end{array}$ \\
\hline 80 & 91 & 68451 & $1 \cdot 80$ & $1.8 \times 10^{-6}$ & 395 & $16 \cdot 9$ & $31 \cdot 5$ \\
\hline 120 & 102 & 54362 & $1 \cdot 84$ & $1.6 \times 10^{-5}$ & 337 & $13 \cdot 3$ & $46 \cdot 3$ \\
\hline
\end{tabular}


To identify the degree of crystallinity in the $\mathrm{Si}: \mathrm{H}$ films prepared from $\mathrm{He}$-diluted $\mathrm{SiH}_{4}$ plasma, X-ray diffraction studies were performed. Figure 3 shows that the films prepared at $P=40 \mathrm{~W}$ do not contain any detectable crystallinity. However, at $P=80 \mathrm{~W}$, the nature of the spectrum changes with prominent diffraction peak at around $2 \theta \sim 28.4^{\circ}$, identifying the development of $\langle 111\rangle$ crystallographic orientations of $\mathrm{Si}$. With farther increase of $P$, the peak intensity corresponding to $\langle 111\rangle$ plane gradually increases and simultaneously two additional prominent peaks originate at around $2 \theta \sim 47.3^{\circ}$ and $\sim 56 \cdot 1^{\circ}$, signifying the simultaneous development of $\langle 220\rangle$ and $\langle 311\rangle$ orientations of $c$-Si within the network structure. The crystalline grain sizes as calculated from the $\mathrm{X}$-ray diffraction peak,

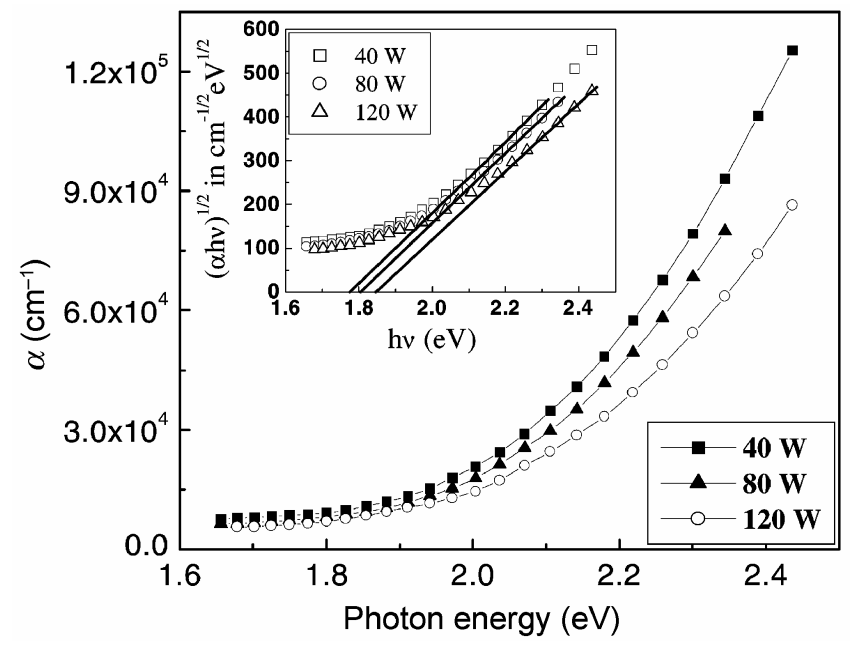

Figure 1. The optical absorption coefficient $(\alpha)$ spectra of the films deposited with different RF power. The inset represents the corresponding Tauc's plot.

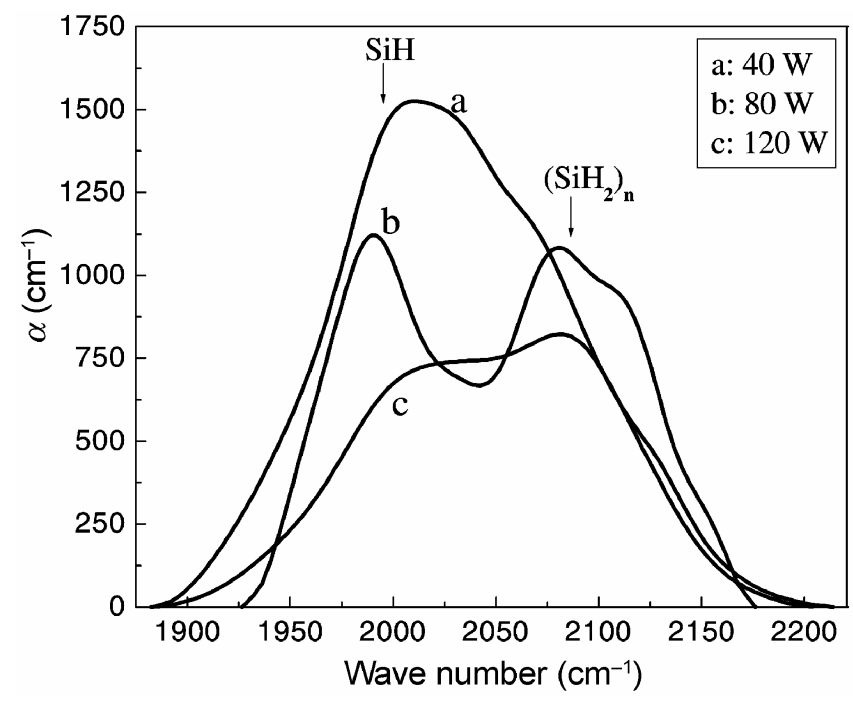

Figure 2. IR absorption spectra in the stretching mode vibration of the samples prepared with different RF power. using Scherrer's formula, are typically $7.7 \mathrm{~nm}$ obtained from $\langle 111\rangle$ planes for $\mathrm{nc}-\mathrm{Si}: \mathrm{H}$ film prepared at RF-power 120 Watt.

To estimate the amount of crystallinity in the network structure, Raman back-scattering studies were performed by a Rainshaw system 2000 micro-Raman spectrometer. The Raman spectrum of the film prepared at $P=40 \mathrm{~W}$ exhibits their amorphous structure by the characteristic broad hump at $480 \mathrm{~cm}^{-1}$, as shown in figure 4 . However, at $P=80 \mathrm{~W}$ prominent crystalline component of the Raman

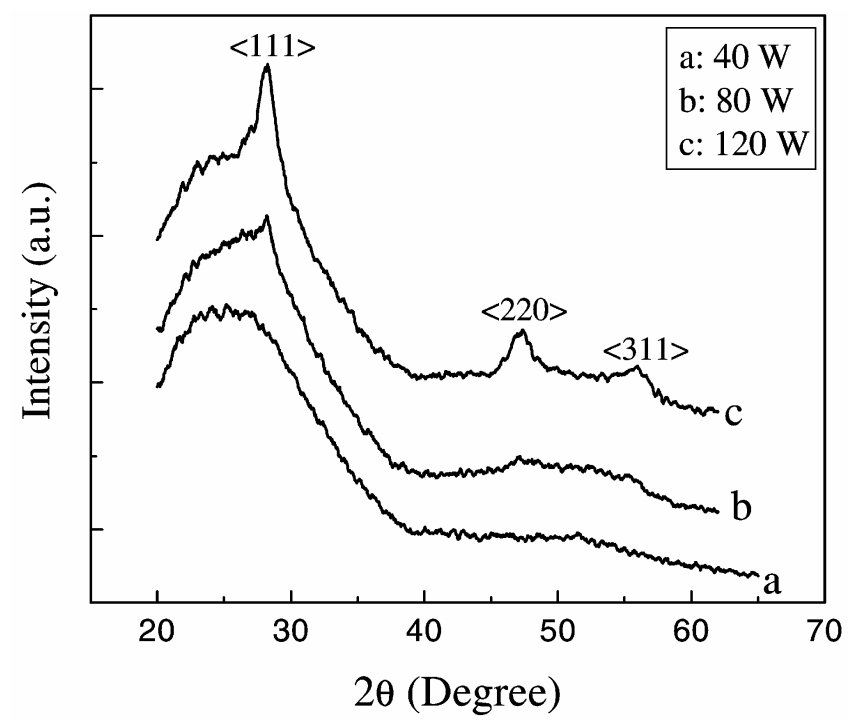

Figure 3. X-ray diffraction pattern of the films deposited with different RF power, exhibiting peaks corresponding to various crystallographic planes.

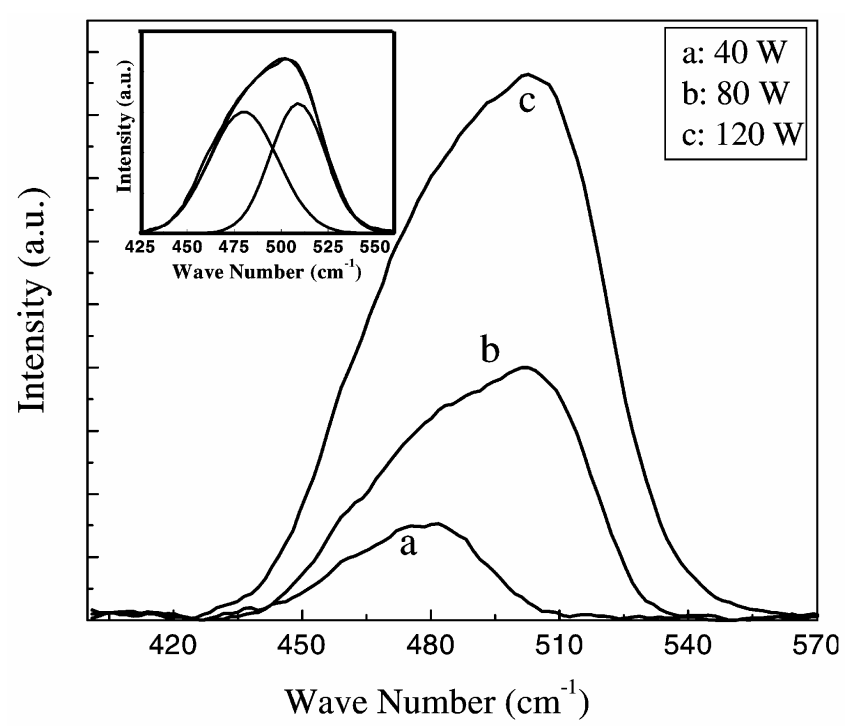

Figure 4. Raman scattering spectra for the samples deposited with different RF power. The inset represents deconvolution of the spectrum for $P=120 \mathrm{~W}$, into corresponding amorphous and crystalline components. 
spectra was observed, with its characteristic peak around $510-520 \mathrm{~cm}^{-1}$. For farther increase in $P$, the peak inten-
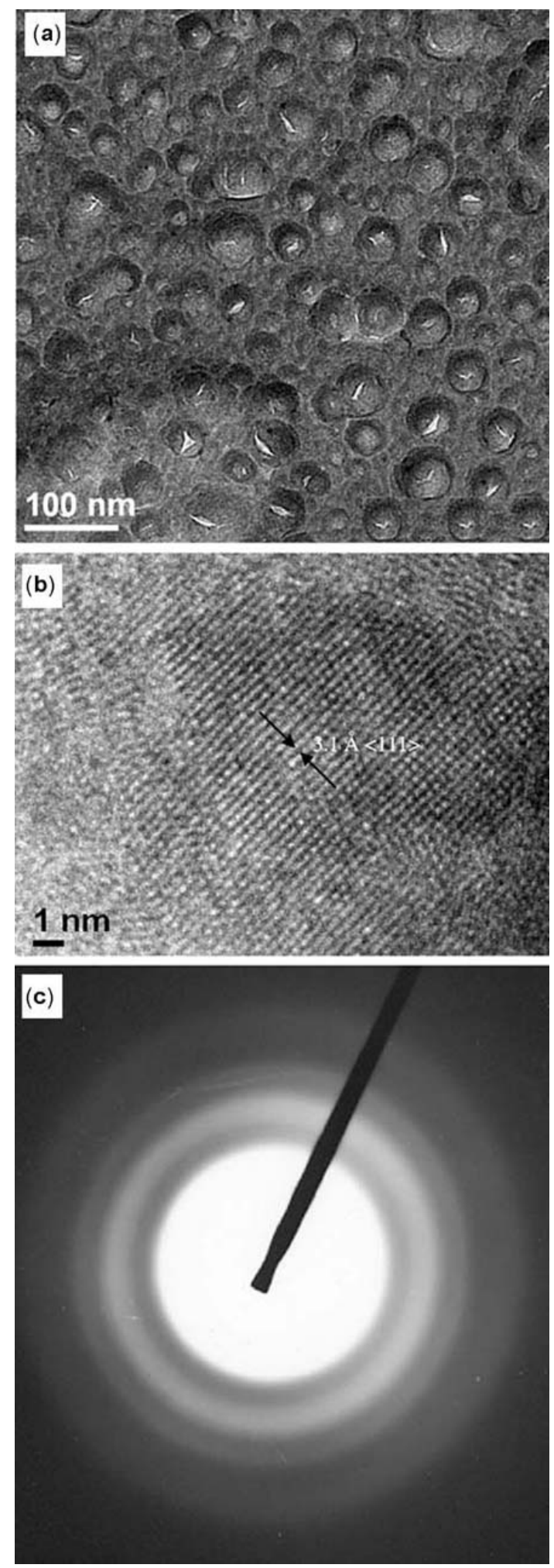

Figure 5. (a) TEM micrograph of the nc-Si:H sample prepared at RF power of $120 \mathrm{~W}$, (b) HRTEM image showing $\langle 111\rangle$ lattice planes for nc-Si : $\mathrm{H}$ film prepared at RF power of $120 \mathrm{~W}$ and (c) electron diffraction pattern for the nc-Si: $\mathrm{H}$ film prepared at RF power of $120 \mathrm{~W}$. sity rapidly increased in general, with its significant contribution around $520 \mathrm{~cm}^{-1}$, identifying very good crystallinity of the film prepared at $P=120 \mathrm{~W}$. The crystalline volume fraction $\left(F_{\mathrm{c}}\right)$ in the network has been estimated using deconvoluted Raman spectra (Das and Bhattacharya 2006) and that has been found to be $\sim 46 \cdot 3 \%$ at $P=120 \mathrm{~W}$, as given in table 1 .

The nanocrystallites have been observed by highresolution transmission electron microscopy using JEOLJSM2010 transmission electron microscope operating at $200 \mathrm{kV}$. Figure 5(a) represents the TEM micrograph of the nc-Si films prepared at $P=120 \mathrm{~W}$. The grain size has been found to vary over a wide range from $10-50 \mathrm{~nm}$, however, much different from that estimated from X-ray diffraction studies. Probably, it could be the clusters of grains those are being depicted by the TEM micrograph while the X-ray diffraction peak width demonstrates the size of individual nanocrystallites. The HRTEM picture (figure 5(b)) exhibits well defined Si lattice planes of mostly $\langle 111\rangle$ crystallographic orientations. Figure 5(c) shows the electron diffraction pattern taken over relatively extended area and that corresponds to the nanocrystalline silicon network of controlled crystalline volume fraction, as estimated from the Raman studies. Similar cluster of grains, as discussed above, are visible from the SEM micrograph also, as shown in figure 6 .

The experimental evidence reveals that the $\mathrm{Si}: \mathrm{H}$ network changes systematically with RF power applied to the plasma. The network maintains a dominantly amorphous structure at $P=40 \mathrm{~W}$. The sample prepared at $P=80 \mathrm{~W}$ corresponds to the onset of crystallinity in $\mathrm{Si}: \mathrm{H}$ network during its growth. The electrical characteristics remains virtually unchanged and the film growth rate increases reasonably, while $\mathrm{Si}-\mathrm{H}$ bonding exhibits a complex heterogeneous structure with almost equal strength of mono-

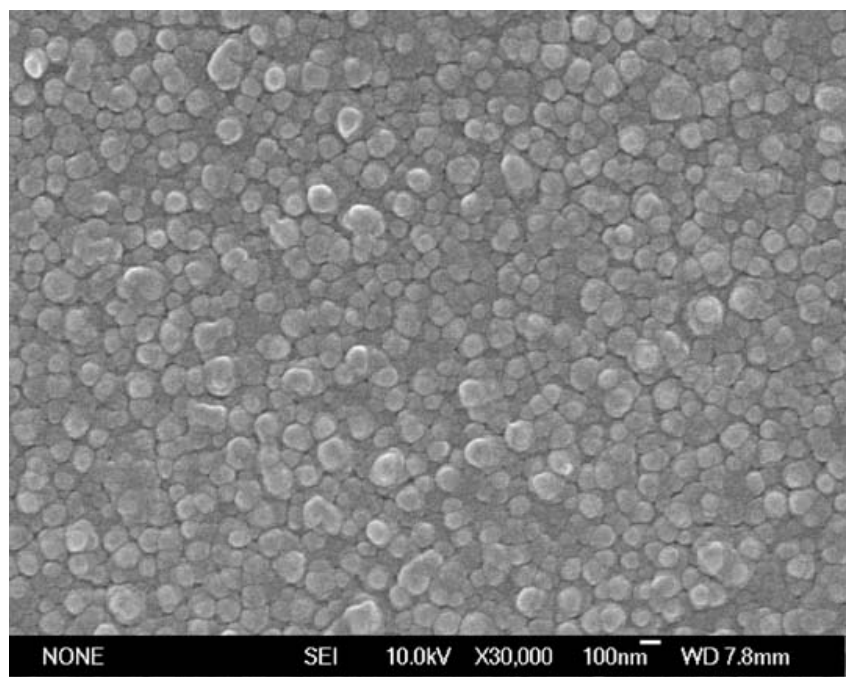

Figure 6. SEM image of the $\mathrm{nc}-\mathrm{Si}: \mathrm{H}$ film prepared at $\mathrm{RF}$ power of $120 \mathrm{~W}$. 
hydride and polyhydride configurations; however, emerging crystallization has been depicted by X-ray diffraction as well as Raman scattering studies. For $P=120 \mathrm{~W}$, sublinear increase in the growth rate $(>100 \AA / \mathrm{min})$ with increasing power, sharp rise in electrical conductivity, optical gap widening, polyhydrogenation of the system with reduced $\mathrm{H}$-content and increasing crystalline volume fraction illustrate noticeable nanocrystallization occurring in $\mathrm{Si}: \mathrm{H}$ network, while HRTEM photograph reveals oriented crystallographic lattice planes, attained by helium dilution to the silane plasma, without using hydrogen, and that is being reported for the first time. Improving crystallinity in nc-Si : $\mathrm{H}$ network at simultaneously enhanced deposition rate could have significant technological impact.

It has been recognized that hydrogen dilution is not essential for the growth of nanocrystalline silicon. In helium diluted silane plasma, energy transfer from metastable $\mathrm{He}^{*}$ state, is directly related to increased dissociation of $\mathrm{SiH}_{4}$, create high-density plasma and enhance the deposition rate. Simultaneously, energy released by the deexcitation of $\mathrm{He}^{*}$ at the growth-zone leads to structural reorientation and promotes crystallization. It has been identified that $\mathrm{He}^{*}$ plays similar role as atomic-H does in the crystallization of $\mathrm{Si}: \mathrm{H}$ network, additionally, high growth rate is attained. By proper optimization of the deposition parameters, nanocrystalline silicon films are attainable at high growth rate from $\mathrm{He}$ diluted $\mathrm{SiH}_{4}$ plasma, without using $\mathrm{H}_{2}$.

\section{Conclusions}

Growth and optimization of the nanocrystalline silicon films have been studied by varying the electrical power applied to the helium diluted silane plasma in RF glow discharge. Wide optical gap and conducting intrinsic nanocrystalline silicon network of controlled crystalline volume fraction and oriented crystallographic lattice planes have been obtained at a reasonably high growth rate from helium diluted silane plasma, without using hydrogen. Improving crystallinity in the network comprising $\sim 10 \mathrm{~nm}$ Si-nanocrystallites and contributing optical gap widening, conductivity ascending and that obtained during simulta- neous escalation of the deposition rate, promises significant technological impact.

\section{Acknowledgements}

The work has been done under the nano-silicon projects funded by the Department of Science and Technology (NSTI scheme) and the Council of Scientific and Industrial Research, Govt. of India.

\section{References}

Das D 1994 Jpn J. Appl. Phys. 33 L571

Das D 2003 J. Phys. D: Appl. Phys. 362335

Das D and Bhattacharya K 2006 J. Appl. Phys. 100103701

Das D, Jana M and Barua A K 2001 J. Appl. Phys. 893041

Dehlinger G, Diehl L, Genser U, Sigg H, Faist J, Ensslin K, Grutzmacher D and Muller E 2000 Science 2902277

Fortunate E, Martins R, Ferreira I, Santos M, Marcario A and Guimaraes I 1989 J. Non-Cryst. Solids 115120

Furukawa S and Miyasato T 1988 Phys. Rev. B38 5726

Jurbergs D, Rogojina E, Mangolini L and Kortshagen U 2006 Appl. Phys. Lett. 88233116

Knights J C 1980 J. Non-Cryst. Solids 35\&36 159

Liu X N, Wu X W, Bao X M and He Y L 1994 Appl. Phys. Lett. 64220

Mangolini L, Thimsen E and Kortshagen U 2005 Nano Lett. 5 655

Matsuda A 1983 J. Non-Cryst. Solids 59\&60 767

Matsuda A, Mashima S, Hasezaki K, Suzuki A, Yamasaki S and McElheny P J 1991 Appl. Phys. Lett. 582494

One $\mathrm{M}$, Saito $\mathrm{M}$, Yoshitomi T, Feigna $\mathrm{C}$, Ohguro $\mathrm{T}$ and Iwai $\mathrm{H}$ 1993 IEDM Tech. Dig. (IEEE), Washington DC p. 119

Prasad K, Finger F, Dubail S, Shah A and Schubert M $1991 J$. Non-Cryst. Solids 137\&138 681

Roca i Cabarrocas P, Nguyen-Tran Th, Djeridane Y, Abramov A, Johnson E and Patriarche G 2007 J. Phys. D40 2258

Sankaran R M, Holunga D, Flagan R C and Giapis K P 2005 Nano Lett. 5537

Takagi H, Ogawa H, Yamasaki Y, Ishizaki A and Nakagiri T 1990 Appl. Phys. Lett. 562379

Tchakarov S, Das D, Saadane O, Kharchenko A V, Suendo V, Kail F and Cabarrocas P Roca i 2004 J. Non-Cryst. Solids 338-340 668

Uchida Y, Ichimura T, Ueno M and Haruki H 1986 Jpn J. Appl. Phys. 21 L586 